\title{
Dispersion relations for mesonic three-body decays
}

\author{
Tobias Isken* \\ Helmholtz-Institut für Strahlen- und Kernphysik (Theorie) and \\ Bethe Center for Theoretical Physics, Universität Bonn, 53115 Bonn, Germany \\ E-mail: isken@hiskp.uni-bonn. de
}

\begin{abstract}
We present dispersive analyses of mesonic three-body decay amplitudes based on the fundamental principles of analyticity, unitarity, and crossing. In this framework the leading final-state interactions are fully taken into account, with the resulting amplitudes being solely dependent on the respective two-body scattering phase shifts of the final-state mesons. The first part focuses on $\eta^{\prime} \rightarrow \eta \pi \pi$ decays. This decay offers several features of interest: due to final-state interactions it can be used to constrain $\pi \eta$ scattering; in the soft-pion limit two Adler zeros are predicted; and the neutral decay channel $\eta^{\prime} \rightarrow \eta \pi^{0} \pi^{0}$ shows a cusp effect within the physical decay region. In the second part we study the quark-mass dependence of $\omega \rightarrow 3 \pi$ decays. We rely on the quark-mass-dependent scattering phase shift for the $\pi \pi P$-wave extracted from unitarized chiral perturbation theory. The described formalism may be used as an extrapolation tool for lattice QCD calculations of three-pion decays, for which $\omega \rightarrow 3 \pi$ can serve as a paradigm case. ${ }^{\dagger}$
\end{abstract}

The 9th International Workshop on Chiral Dynamics

17-21 September 2018

Durham, NC, USA

\footnotetext{
* Speaker.

${ }^{\dagger}$ These proceedings draw heavily from two published papers [1,2].
} 


\section{Introduction}

The decay $\eta^{\prime} \rightarrow \eta \pi \pi$ has received considerable interest in past years for several reasons. Due to the $\mathrm{U}(1)_{A}$ anomaly the $\eta^{\prime}$ is not a Goldstone boson and therefore cannot be described in "standard" chiral perturbation theory (ChPT). Hence, extended versions like large- $N_{c}$ ChPT and resonance chiral theory (RChT) are needed to account for interactions involving the $\eta^{\prime}[3,4]$. Experimental measurements of the Dalitz plot of the charged channel have been performed by VES [5] and BES-III [6,7], for the neutral channel by the GAMS-4 $\pi$ [8], A2 [9], and BES-III [7] collaborations. Since the mass of the $\eta^{\prime}$ is still sufficiently small so that $\eta^{\prime} \rightarrow \eta \pi \pi$ decays are not polluted by nonvirtual $K \bar{K}$ intermediate states, it could be used to constrain $\pi \eta$ scattering. Furthermore, the $\eta^{\prime} \rightarrow \eta \pi^{0} \pi^{0}$ decay channel is expected to show a cusp effect at the charged-pion threshold [10], similar to what has been studied extensively for $K \rightarrow 3 \pi$ decays (see Ref. [11] and references therein). This phenomenon has recently been confirmed by the A2 collaboration [9]. In the following we use a dispersion-theoretical approach to study $\eta^{\prime} \rightarrow \eta \pi \pi$ decays.

Decays of light isoscalar vector mesons into three pions are an ideal paradigm case for testing dispersion relations. Due to Bose symmetry only odd partial waves are allowed to contribute, so neglecting $F$ - and higher partial wave discontinuities, the decay is fully described by the $P$-wave $\pi \pi$ amplitudes. The dispersive framework to describe this decay was already used in previous studies of $\omega / \phi \rightarrow 3 \pi[12,13]$ as well as the related processes $\gamma^{*} \pi \rightarrow \pi \pi$ [14-19]. Here we want to assess the quark-mass dependence of the $\omega \rightarrow 3 \pi$ decay amplitude based on dispersion relations, which requires the quark-mass dependent $\pi \pi$ phase shift as input. While we still need to rely on effective-field-theory ideas to describe the variation of the $\omega$ mass with the quark masses, the dispersive framework allows us to predict its quark-mass-dependent width. The idea to employ dispersion theory to extend the applicability of quark-mass-dependent phase shifts is not new: it has already been applied to describe the pion vector form factor [20], as well as, in a formalism closely related to what we present here, to the reaction $\gamma^{*} \pi \rightarrow \pi \pi[19]$.

\section{Dispersion relations for $\eta^{\prime} \rightarrow \eta \pi \pi$}

The dispersion relations for the decay $\eta^{\prime} \rightarrow \eta \pi \pi$ are set up in analogy to previous work on different decays into three pions [12,21-23]. The amplitude is decomposed in terms of functions of one Mandelstam variable only that only possess a right hand cut, which leads to the form

$$
\mathscr{M}(s, t, u)=\mathscr{M}_{0}(s)+\mathscr{M}_{1}(t)+\mathscr{M}_{1}(u) .
$$

Here, $\mathscr{M}_{I}$ refers to functions of isospin $I$ : isospin conservation constrains the total isospin of the final-state pion pair to $I=0$, while the $\pi \eta$ system has $I=1$. Equation (2.1) follows from a partialwave expansion of the discontinuities in fixed- $s$, $-t$, and $-u$ dispersion relations when restricted to $S$-waves. The unitarity relations for the single-variable functions $\mathscr{M}_{I}$ along the right-hand cut are given by

$$
\operatorname{disc} \mathscr{M}_{I}(s)=2 i \theta\left(s-s_{\mathrm{thr}}\right)\left[\mathscr{M}_{I}(s)+\hat{\mathscr{M}}_{I}(s)\right] \sin \delta_{I}(s) e^{-i \delta_{I}(s)},
$$

where the $\theta$-function ensures the correct opening at the respective threshold $s_{\mathrm{thr}}$, i.e. $4 M_{\pi}^{2}$ for the $\pi \pi$ and $\left(M_{\eta}+M_{\pi}\right)^{2}$ for the $\pi \eta$ channel. Below the opening of inelastic channels, $\delta_{I}(s)$ agrees with the 

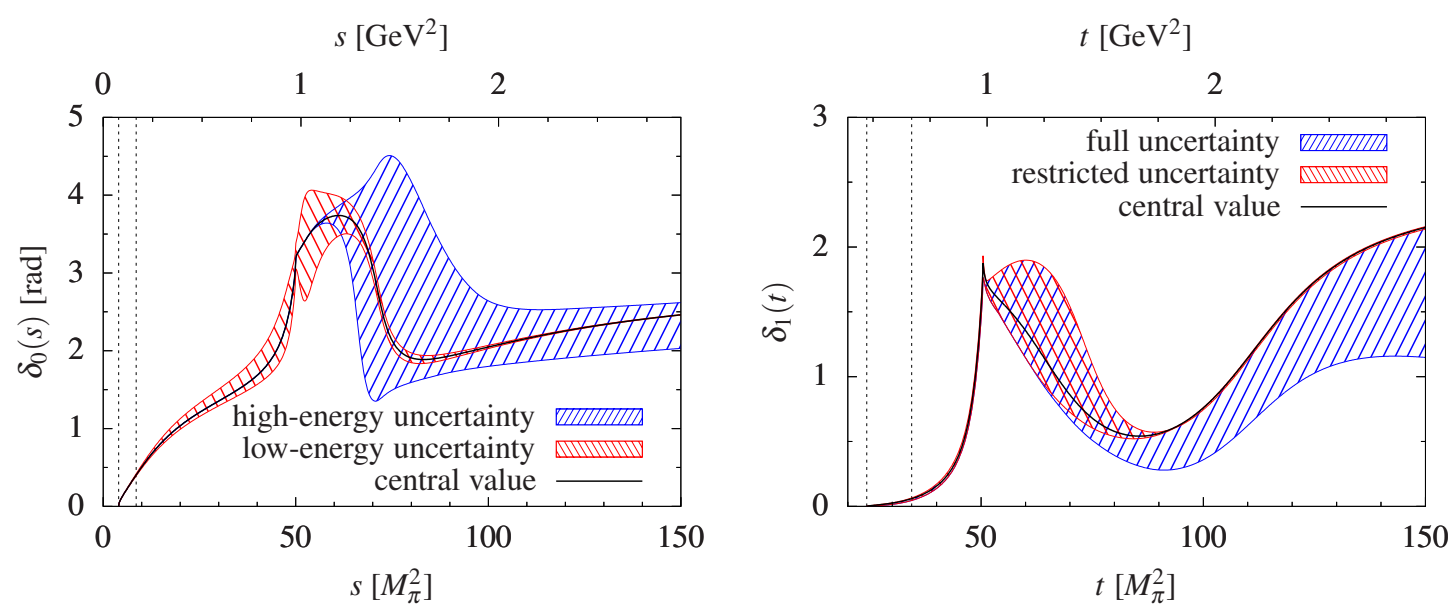

Figure 1: Left panel: the isospin-zero $\pi \pi S$-wave effective phase shift $\delta_{0}(s)$, constructed with input from [26,27]. Right panel: the $\pi \eta S$-wave effective phase shift $\delta_{1}(t)$ [28]. The boundaries of the allowed physical region of the decay $\eta^{\prime} \rightarrow \eta \pi \pi$ are indicated by dashed vertical lines. Figures adapted from Ref. [1].

phase of elastic $\pi \pi$ or $\pi \eta$ scattering due to Watson's final-state theorem [24]. The inhomogeneities $\hat{\mathscr{M}}_{I}$ are given by

$$
\hat{\mathscr{M}}_{0}(s)=\int_{-1}^{1} \mathrm{~d} z_{s} \mathscr{M}_{1}\left(t\left(s, z_{s}\right)\right), \quad \hat{\mathscr{M}}_{1}(t)=\frac{1}{2} \int_{-1}^{1} \mathrm{~d} z_{t}\left[\mathscr{M}_{0}\left(s\left(t, z_{t}\right)\right)+\mathscr{M}_{1}\left(u\left(t, z_{t}\right)\right)\right],
$$

where $z_{s, t}$ define the cosine of the scattering angles in the $s$ - and $t$-channel. Note that the analytic continuation of Eq. (2.3) both in the Mandelstam variables and the decay mass $M_{\eta^{\prime}}$ involves several subtleties, for further details see Ref. [25].

The crucial input in the dispersion relation consists of the $\pi \pi$ and $\pi \eta S$-wave phase shifts $\delta_{0}$ and $\delta_{1}$ shown in Fig. 1. In order to take inelastic $K \bar{K}$ effects into account, while still solving a single-channel dispersive problem (2.2) only, we resort to the construction of effective phase shifts. Below the $K \bar{K}$ threshold our phase $\delta_{0}$ agrees with the Roy solution [26]. In the inelastic regime we continue the phase via a unitarized large- $N_{c}$ ChPT prediction for $\eta^{\prime} \eta \rightarrow \pi \pi / K \bar{K}$ using a coupledchannel Omnès matrix [27]. This treatment generates a smooth phase drop by $\pi$ with respect to the elastic $\pi \pi$ scattering phase. For the $\pi \eta$ phase shift $\delta_{1}$, we take the phase of the scalar form factor $F_{S}^{\pi \eta}$ extracted from a $\pi \eta / K \bar{K}$ coupled-channel $T$-matrix [28] as input.

Assuming that the amplitude scales like a constant in the high-energy limit, we can write the solutions of the unitarity relations Eq. (2.2) as

$$
\begin{aligned}
& \mathscr{M}_{0}(s)=\Omega_{0}(s)\left\{\alpha+\beta s+\frac{s^{2}}{\pi} \int_{s_{\mathrm{thr}}}^{\infty} \frac{\mathrm{d} s^{\prime}}{s^{\prime 2}} \frac{\hat{\mathscr{M}}_{0}\left(s^{\prime}\right) \sin \delta_{0}\left(s^{\prime}\right)}{\left|\Omega_{0}\left(s^{\prime}\right)\right|\left(s^{\prime}-s\right)}\right\}, \\
& \mathscr{M}_{1}(t)=\Omega_{1}(t)\left\{\gamma t+\frac{t^{2}}{\pi} \int_{t_{\mathrm{thr}}}^{\infty} \frac{\mathrm{d} t^{\prime}}{t^{\prime 2}} \frac{\hat{\mathscr{M}}_{1}\left(t^{\prime}\right) \sin \delta_{1}\left(t^{\prime}\right)}{\left|\Omega_{1}\left(t^{\prime}\right)\right|\left(t^{\prime}-t\right)}\right\},
\end{aligned}
$$

with three subtraction constants $\alpha, \beta$, and $\gamma$. Here $\Omega_{I}$ denotes the Omnès function [29] corresponding to the phase $\delta_{I}$. After solving the system of coupled integral equations Eqs. (2.3) and (2.4) with an iterative numerical procedure, we have to determine the free parameters in the dispersion relation, i.e. the subtraction constants $\alpha, \beta$, and $\gamma$. In experimental analyses of the $\eta^{\prime} \rightarrow \eta \pi \pi$ Dalitz 

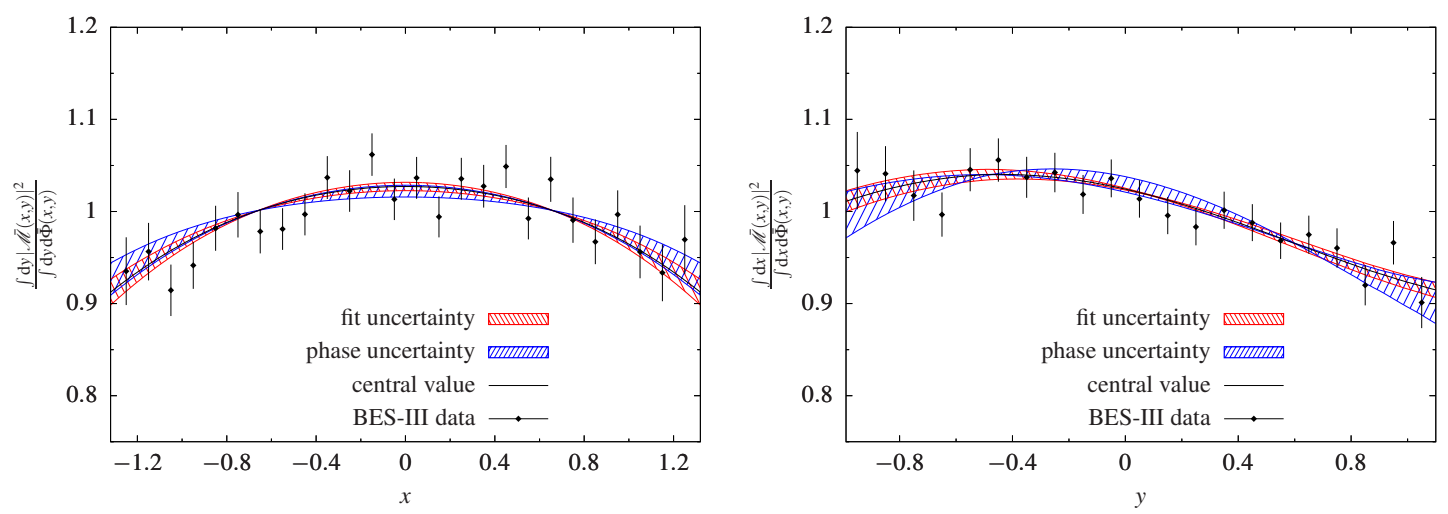

Figure 2: Decay spectra for $\eta^{\prime} \rightarrow \eta \pi^{+} \pi^{-}$divided by the phase space $\mathrm{d} \bar{\Phi}(x, y)$, where both are individually normalized. Left panel: integrated over the $y$-direction. Right panel: integrated over the $x$-direction. Figures adapted from Ref. [1].

plot, the squared amplitude is expanded in terms of symmetrized coordinates $x$ and $y$ according to

$$
\left|\mathscr{M}_{\exp }(x, y)\right|^{2}=\left|\mathscr{N}_{\exp }\right|^{2}\left\{1+a y+b y^{2}+d x^{2}+\ldots\right\},
$$

and the parameters $a, b, d$ are fitted to experimental data $(x \propto(t-u)$ and $y$ is linear in $s$, see [1]). For our analysis, we have generated a pseudodata sample from the $\eta^{\prime} \rightarrow \eta \pi^{+} \pi^{-}$Dalitz-plot distribution [30] as measured by the BES-III collaboration [6]. The overall normalization of the amplitude is constrained by the partial decay width [31].

Fitting real subtraction constants to the data yields $\chi^{2} /$ ndof $=459 / 435 \approx 1.06$. In Fig. 2 , we display the decay spectrum integrated over the Dalitz-plot variables $x$ or $y$, respectively. The statistical "fit uncertainty" is dominated by the experimental uncertainty in the Dalitz-plot distribution, while the uncertainty due to the partial decay width is small. The very asymmetric systematic "phase uncertainty" combines the error of the $\pi \pi$ and $\pi \eta$ phase input, which is mainly generated by the $\pi \eta$ phase variation at high energies.

In the limit of one of the pion momenta going to zero, current algebra predicts two Adler zeros of the amplitude [32-34] protected by $\mathrm{SU}(2) \times \mathrm{SU}(2)$ symmetry. The soft-pion theorem implies zeros of the on-shell amplitude at the two soft-pion points

$$
s_{1}=s_{2}=2 M_{\pi}^{2}, \quad t_{1}=u_{2}=M_{\eta^{\prime}}^{2}, \quad u_{1}=t_{2}=M_{\eta}^{2} .
$$

In the past, claims have been made that the $a_{0}(980)$ resonance removes the Adler zeros based on the explicit inclusion of a scalar resonance propagator [35]. In Fig. 3, we show the result for the dispersive amplitude fitted to data, evaluated along a line of fixed $s=2 M_{\pi}^{2}$. We encounter zeros in both the real and imaginary part of the amplitude at positions close to the soft-pion points, but for slightly smaller values of $|t-u|$. At the resonance positions

$$
|t-u| \approx 2 M_{a_{0}}^{2}-M_{\eta^{\prime}}^{2}-M_{\eta}^{2},
$$

which are also close but outside the soft-pion points, we observe a large peak in the imaginary part and another zero in the real part. We conclude that the dispersive representation refutes the argument [35] that for $\eta^{\prime} \rightarrow \eta \pi \pi$ the low-energy theorem does not result in an Adler zero. Although 


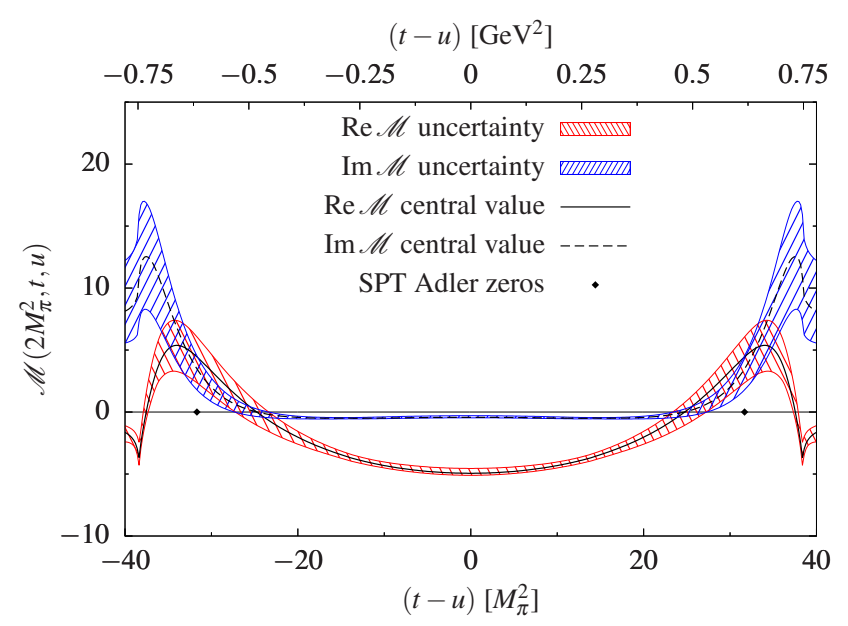

Figure 3: Real and imaginary part of the amplitude along a line of fixed $s=2 M_{\pi}^{2}$. The subtraction constants of the dispersive representation are fixed by a fit to the BES-III data set. Figure adapted from Ref. [1].

the corrections at the soft-pion points are of $\mathscr{O}\left(M_{\pi}^{2} /\left(M_{\eta^{\prime}}^{2}-M_{a_{0}}^{2}\right)\right)$, which is not a small quantity, the zeros of the amplitude survive and are just shifted to smaller values of $|t-u|$.

So far, we have analyzed experimental Dalitz-plot data sets for $\eta^{\prime} \rightarrow \eta \pi^{+} \pi^{-}$. To deduce a comparably precise prediction for the neutral final state $\eta^{\prime} \rightarrow \eta \pi^{0} \pi^{0}$, we have to consider potentially enhanced sources of isospin-symmetry violation. While a correction for phase space alone is straightforward, we need to construct phase shift input that has all the thresholds in the right places. We observe that the cusp structure of the decay amplitude for $\eta^{\prime} \rightarrow \eta \pi^{0} \pi^{0}$ [10] is very similar to that of the neutral-pion scalar form factor $F_{0}(s)$ [36]. Thus, we employ $\arg F_{0}(s)$ as the input $\pi^{0} \pi^{0}$ $S$-wave phase shift, see Fig. 4 . In the $\pi \eta$ case we rely on a simple rescaling to adapt the $\pi^{ \pm} \eta$ phase shift to $\pi^{0} \eta$ in such a way as to put all thresholds into the right places.

Our prediction for the $\eta^{\prime} \rightarrow \eta \pi^{0} \pi^{0}$ decay spectrum projected on the $y$ direction is shown in Fig. 4, where the nonanalytic structure of the $\pi^{+} \pi^{-}$cusp is clearly visible. It is based on the
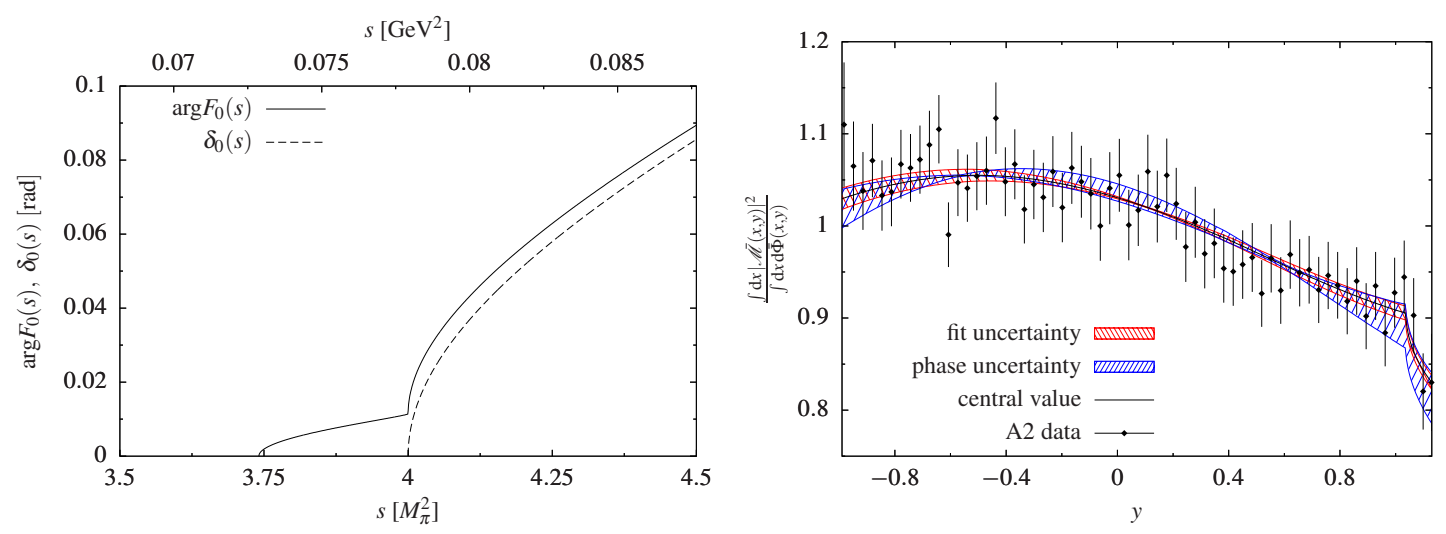

Figure 4: Left panel: comparison of $\arg F_{0}(s)$ [36] (solid line) to the isospin-symmetric phase $\delta_{0}(s)$ [26] (dashed line). Figure adapted from Ref. [1]. Right panel: decay spectrum for $\eta^{\prime} \rightarrow \eta \pi^{0} \pi^{0}$ integrated over the variable $x$ and divided by the phase space $\mathrm{d} \bar{\Phi}(x, y)$, where both are individually normalized. For comparison, we plot the data sample of Analysis II from the A2 collaboration [9]. 
subtraction constants as extracted from the BES-III fit to the $\eta^{\prime} \rightarrow \eta \pi^{+} \pi^{-}$channel. As the figure shows, the predicted spectrum is in very good agreement with the experimental results from A2 [9].

As a further theoretical development, the fitted dispersive parametrization of $\eta^{\prime} \rightarrow \eta \pi \pi$ will be used as an input in a forthcoming analysis of inelasticity effects in $\eta^{\prime} \rightarrow 3 \pi$ [37]. Notice that the decay $\eta^{\prime} \rightarrow 3 \pi$ can proceed via $\eta^{\prime} \rightarrow \eta \pi \pi$ and isospin-breaking rescattering $\eta \pi \rightarrow \pi \pi$ (which can be extracted from analytic continuation of the dispersive amplitude $\eta \rightarrow 3 \pi$ [38]) and direct isospin breaking $\eta^{\prime} \rightarrow 3 \pi$.

\section{Quark-mass dependence of $\omega \rightarrow 3 \pi$}

Many studies of complicated observables within lattice QCD are still performed with light quarks that are heavier than they are in the real world (see e.g. Refs. [39, 40] for reviews). To extrapolate such simulations to the physical point, additional theoretical input is required. At low energies, the effective field theory that controls the quark-mass dependence by construction is ChPT [41-43], which describes the interactions of the pseudoscalar octet $(\pi, K, \eta)$. In ChPT the pion mass is directly related to the light quark mass [44], hence we will refer to the pion-mass dependence instead. The vast majority of states in QCD are resonances, and to perform chiral extrapolations for these is less straightforward. However, for the $\rho$ in $P$-wave $\pi \pi \rightarrow \pi \pi$ scattering we can derive the pion-mass dependence of the complete pole position by means unitarized versions of ChPT, such as the inverse amplitude method (IAM) [45-48]. In contrast for the $\omega$, appearing in $3 \pi \rightarrow 3 \pi$ scattering of the appropriate quantum numbers, we are not in the position to do the same.

We will mainly discuss the complicated pion-mass dependence of the width of the $\omega$, or the imaginary part of its pole in the complex plane, in the following; for the pion-mass dependence of its mass, the corresponding real part, we have to resort to symmetry arguments based on SU(3) effective Lagrangians [49-51]. We find that SU(3) breaking effects do not significantly affect the relation between $M_{\rho}$ and $M_{\omega}$, hence we adopt the pion-mass dependence of $M_{\rho}\left(M_{\pi}^{2}\right)$ for $M_{\omega}\left(M_{\pi}^{2}\right)$.

The process $\omega \rightarrow 3 \pi$ gives the by far dominant contribution to the total $\omega$ decay width; in this study we assume the latter to be fully saturated by this dominant decay channel. By integrating the squared amplitude over phase space we obtain the decay width $\Gamma(\omega \rightarrow 3 \pi) \equiv \Gamma_{\omega}$ according to

$$
\Gamma_{\omega}=\frac{1}{256 \pi^{3} M_{\omega}^{3}} \int \mathrm{d} s \mathrm{~d} t|\mathscr{M}(s, t, u)|^{2} .
$$

Since the transition $\omega \rightarrow 3 \pi$ is of odd intrinsic parity, the modulus of the amplitude can be decomposed into a kinematic prefactor and a scalar function $\mathscr{F}$ containing the dynamical information,

$$
|\mathscr{M}(s, t, u)|^{2}=\frac{1}{4}\left[s t u-M_{\pi}^{2}\left(M_{\omega}^{2}-M_{\pi}^{2}\right)^{2}\right]|\mathscr{F}(s, t, u)|^{2} .
$$

Due to Bose symmetry only odd partial waves are allowed to contribute to the process. Neglecting discontinuities from $F$ - and higher partial waves allows us to decompose the scalar function into a sum of single-variable functions with definite isospin $I=1[12-16,18]$

$$
\mathscr{F}(s, t, u)=\mathscr{F}_{1}(s)+\mathscr{F}_{1}(t)+\mathscr{F}_{1}(u),
$$



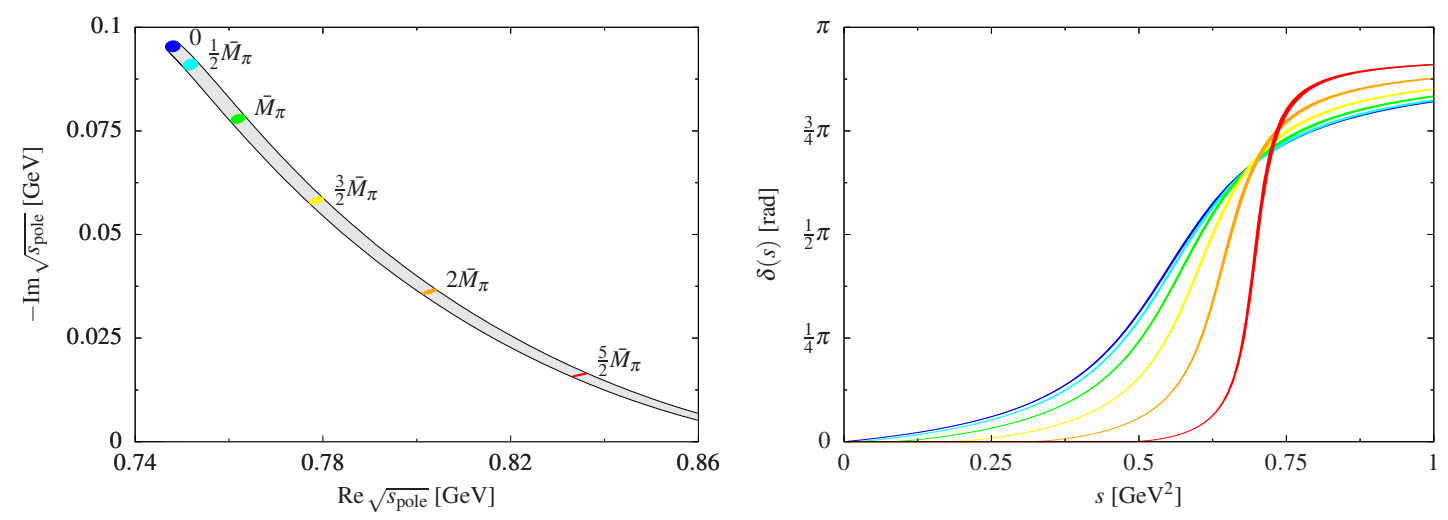

Figure 5: Left panel: trajectory of the $\rho$ pole position in the complex $s$ plane for different pion masses. The colored ellipses mark the one- $\sigma$ uncertainty regions of the pole position for the respective pion mass. Right panel: IAM amplitude $\pi \pi P$-wave scattering phase shift for different values of the pion mass: 0 (blue), $\frac{1}{2} \bar{M}_{\pi}$ (cyan), $\bar{M}_{\pi}$ (green), $\frac{3}{2} \bar{M}_{\pi}$ (yellow), $2 \bar{M}_{\pi}$ (orange), and $\frac{5}{2} \bar{M}_{\pi}$ (red). The error bands result from the uncertainty of $F$ and $\bar{l}$. Here $\bar{M}_{\pi}$ denotes the physical value of the pion mass. Figures taken from Ref. [2].

where $\mathscr{F}_{1}$ possesses only a right-hand cut (see Ref. [12] for a discussion of potential $F$-wave contributions). The single-variable functions fulfill the unitarity condition

$$
\operatorname{disc} \mathscr{F}_{1}(s)=2 i \theta\left(s-4 M_{\pi}^{2}\right)\left[\mathscr{F}_{1}(s)+\hat{\mathscr{F}}_{1}(s)\right] \sin \delta(s) e^{-i \delta(s)},
$$

where the inhomogeneity $\hat{\mathscr{F}}_{1}$ is defined as an angular integral according to

$$
\hat{\mathscr{F}}_{1}(s)=\frac{3}{2} \int_{-1}^{1} \mathrm{~d} z_{s}\left(1-z_{s}^{2}\right) \mathscr{F}_{1}\left(t\left(s, z_{s}\right)\right) .
$$

We treat the final-state $\pi \pi$ rescattering to be elastic, thus it can be described in terms of the $\pi \pi P$-wave phase shift $\delta(s)$ only. Using the IAM technique to unitarize SU(2) ChPT we construct a pion-mass-dependent $P$-wave scattering amplitude [52,53]. At one loop this amplitude depends only on three parameters: the pion mass, the pion decay constant in the chiral limit $F$, and one linear combination of low-energy constants $\bar{l}$. Note that both $F$ as well as $\bar{l}$ are independent of $M_{\pi}$.

The trajectory of the pole position of the $\rho$ resonance as well as the corresponding $\pi \pi$ scattering phase shift are displayed in Fig. 5. As expected from its quark content, the mass of the $\rho$ increases if the pion becomes heavier. This behavior can be described to good approximation as a linear function in $M_{\pi}^{2}$. For heavier pions the slope of the phase shift becomes steeper, while the whole curve moves to the right (decreasing width and increasing mass of the $\rho$ ). This observation is also reported by various lattice QCD calculations carried out at different pion masses [54-65]. In the low-energy regime at the physical pion mass the phase shift is in perfect agreement with the Roy analyses of Refs. [26, 66].

We employ a solution of the unitarity relation (3.4) with a single subtraction constant $\alpha$ [12],

$$
\mathscr{F}_{1}(s)=\Omega(s)\left\{\alpha+\frac{s}{\pi} \int_{4 M_{\pi}^{2}}^{\infty} \frac{\mathrm{d} s^{\prime}}{s^{\prime}} \frac{\hat{\mathscr{F}}_{1}\left(s^{\prime}\right) \sin \delta\left(s^{\prime}\right)}{\left|\Omega\left(s^{\prime}\right)\right|\left(s^{\prime}-s\right)}\right\} .
$$

Based on vector-meson-dominance arguments, we treat $\alpha$ as pion-mass independent. As $\alpha$ serves as an overall normalization, at physical pion masses it is fixed to the decay rate $\Gamma_{\omega}$, with the energy dependence of the amplitude or the Dalitz plot distribution then being a theoretical prediction [12]. 

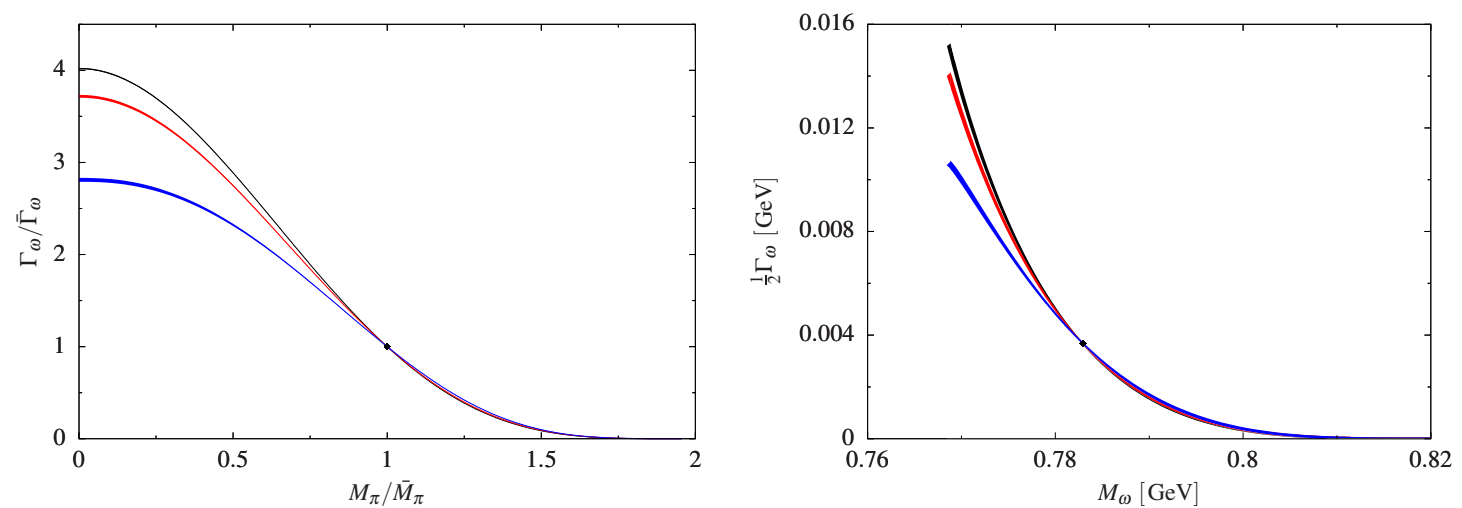

Figure 6: Left panel: pion-mass-dependent decay width for $\omega \rightarrow 3 \pi$. $\bar{\Gamma}_{\omega}$ and $\bar{M}_{\pi}$ denote values at the physical point. Right panel: trajectory of the pion-mass-dependent $\omega$ mass and width in the complex-energy plane of $3 \pi \rightarrow 3 \pi$ scattering. Both plots show: kinematics only (black), 2-body dynamics (red), and full 3-body dynamics (blue). The physical point is marked by the black diamond. The error bands are generated by taking the uncertainties of the IAM phase shift and $M_{\omega}\left(M_{\pi}^{2}\right)$ into account. Figures taken from Ref. [2].

In order to study the dynamical effects on the decay width we consider three different scenarios: first we consider only kinematic effects on the decay width (all pion-pion dynamics are disregarded, $\delta(s)=0$, and thus $|\mathscr{F}(s, t, u)|^{2}=$ const.); second we allow for 2-body rescattering effects (meaning $|\mathscr{F}(s, t, u)|^{2} \propto|\Omega(s)+\Omega(t)+\Omega(u)|^{2}$ ); and third the full 3-body dynamics are taken into account. A comparison of the different cases is displayed in Fig. 6.

Since the mass of the three pions is increasing faster than the mass of the $\omega$, the $\omega \rightarrow 3 \pi$ width decreases with increasing pion mass. At 1.96 physical pion masses, the masses of the three pions exceed the $\omega$ mass, thus the decay $\omega \rightarrow 3 \pi$ is no longer allowed and the $\omega$ becomes stable with respect to the considered decay channel (i.e., in QCD in the isospin limit).

The effects of the 2- and 3-body dynamics are strongly weighted by the kinematical prefactor (3.2), which vanishes at the phase space boundaries in all directions. Since the dynamics are largely driven by the $\rho$ resonance and the $\omega \rightarrow 3 \pi$ phase space tightens for increasing pion mass, we conclude that strong imprints of the $\rho$ will only affect the width close to the chiral limit. Approaching the physical point the influence of the dynamical effects become weaker. Beyond the physical point the curves of 2- and 3-body dynamics do not differ strongly from the curve of pure kinematics.

Using this result we can predict a pion-mass-dependent trajectory of the $\omega$ pole position in the complex-energy plane of $3 \pi \rightarrow 3 \pi$ scattering Fig. 6, in analogy to the $\rho$ in Fig. 5 .

Since dynamical effects in $\omega \rightarrow 3 \pi$ are limited due to the small phase space, we want to emphasize that the dispersive representation in Eq. (3.6) is valid for general $V \rightarrow 3 \pi$ decays, with $V$ denoting an arbitrary isoscalar vector meson, at least to the extent that the elastic approximation in the $\pi \pi$ rescattering is justifiable; see Ref. [2] for an explicit discussion of $\phi \rightarrow 3 \pi$.

\section{Acknowledgments}

Partial financial support by DFG and NSFC through funds provided to the Sino-German CRC 110 "Symmetries and the Emergence of Structure in QCD" (DFG Grant No. TRR110 and NSFC Grant No. 11621131001) is gratefully acknowledged. 


\section{References}

[1] T. Isken, B. Kubis, S. P. Schneider, and P. Stoffer, Eur. Phys. J. C 77, 489 (2017).

[2] M. Dax, T. Isken, and B. Kubis, Eur. Phys. J. C 78, 859 (2018).

[3] R. Escribano, P. Masjuan, and J. J. Sanz-Cillero, JHEP 05, 094 (2011).

[4] S. Gonzàlez-Solís and E. Passemar, Eur. Phys. J. C 78, 758 (2018).

[5] V. Dorofeev et al., Phys. Lett. B 651, 22 (2007).

[6] M. Ablikim et al. [BESIII Collaboration], Phys. Rev. D 83, 012003 (2011).

[7] M. Ablikim et al. [BESIII Collaboration], Phys. Rev. D 97, 012003 (2018).

[8] A. M. Blik et al., Phys. Atom. Nucl. 72, 231 (2009).

[9] P. Adlarson et al., Phys. Rev. D 98, 012001 (2018).

[10] B. Kubis and S. P. Schneider, Eur. Phys. J. C 62, 511 (2009).

[11] J. Gasser, B. Kubis, and A. Rusetsky, Nucl. Phys. B 850, 96 (2011).

[12] F. Niecknig, B. Kubis, and S. P. Schneider, Eur. Phys. J. C 72, 2014 (2012).

[13] I. V. Danilkin et al., Phys. Rev. D 91, 094029 (2015).

[14] M. Hoferichter, B. Kubis, and D. Sakkas, Phys. Rev. D 86, 116009 (2012).

[15] M. Hoferichter et al., Eur. Phys. J. C 74, 3180 (2014).

[16] M. Hoferichter, B. Kubis, and M. Zanke, Phys. Rev. D 96, 114016 (2017).

[17] M. Hoferichter et al., Phys. Rev. Lett. 121, 112002 (2018).

[18] M. Hoferichter et al., JHEP 10, 141 (2018).

[19] M. Niehus, M. Hoferichter, and B. Kubis, PoS CD2018, 076 (2019).

[20] F.-K. Guo, C. Hanhart, F. J. Llanes-Estrada, and U.-G. Meißner, Phys. Lett. B 678, 90 (2009).

[21] N. N. Khuri and S. B. Treiman, Phys. Rev. 119, 1115 (1960).

[22] A. V. Anisovich and H. Leutwyler, Phys. Lett. B 375, 335 (1996).

[23] J. Kambor, C. Wiesendanger, and D. Wyler, Nucl. Phys. B 465, 215 (1996).

[24] K. M. Watson, Phys. Rev. 95, 228 (1954).

[25] S. P. Schneider, PhD thesis, Bonn U. (2012).

[26] I. Caprini, G. Colangelo, and H. Leutwyler, Eur. Phys. J. C 72, 1860 (2012).

[27] J. T. Daub, C. Hanhart, and B. Kubis, JHEP 02, 009 (2016).

[28] M. Albaladejo and B. Moussallam, Eur. Phys. J. C 75, 488 (2015).

[29] R. Omnès, Nuovo Cim. 8, 316 (1958).

[30] A. Kupść, private communication.

[31] M. Tanabashi et al. [Particle Data Group], Phys. Rev. D 98, 030001 (2018).

[32] Riazuddin and S. Oneda, Phys. Rev. Lett. 27, 548 (1971).

[33] S. L. Adler, Phys. Rev. 137, B1022 (1965). 
[34] S. L. Adler, Phys. Rev. 139, B1638 (1965).

[35] N. G. Deshpande and T. N. Truong, Phys. Rev. Lett. 41, 1579 (1978).

[36] G. Colangelo, J. Gasser, and A. Rusetsky, Eur. Phys. J. C 59, 777 (2009).

[37] T. Isken, B. Kubis, and P. Stoffer, in preparation.

[38] S. Descotes-Genon and B. Moussallam, Eur. Phys. J. C 74, 2946 (2014).

[39] S. Aoki et al., Eur. Phys. J. C 77, 112 (2017).

[40] R. A. Briceño, J. J. Dudek, and R. D. Young, Rev. Mod. Phys. 90, 025001 (2018).

[41] S. Weinberg, Physica A 96, 327 (1979).

[42] J. Gasser and H. Leutwyler, Annals Phys. 158, 142 (1984).

[43] J. Gasser and H. Leutwyler, Nucl. Phys. B 250, 465 (1985).

[44] M. Gell-Mann, R. J. Oakes, and B. Renner, Phys. Rev. 175, 2195 (1968).

[45] T. N. Truong, Phys. Rev. Lett. 67, 2260 (1991).

[46] A. Dobado and J. R. Peláez, Phys. Rev. D 47, 4883 (1993).

[47] A. Dobado and J. R. Peláez, Phys. Rev. D 56, 3057 (1997).

[48] A. Gómez Nicola, J. R. Peláez, and G. Ríos, Phys. Rev. D 77, 056006 (2008).

[49] E. E. Jenkins, A. V. Manohar, and M. B. Wise, Phys. Rev. Lett. 75, 2272 (1995).

[50] J. Bijnens and P. Gosdzinsky, Phys. Lett. B 388, 203 (1996).

[51] J. Bijnens, P. Gosdzinsky, and P. Talavera, Nucl. Phys. B 501, 495 (1997).

[52] C. Hanhart, J. R. Peláez, and G. Ríos, Phys. Rev. Lett. 100, 152001 (2008).

[53] J. R. Peláez and G. Ríos, Phys. Rev. D 82, 114002 (2010).

[54] S. Aoki et al. [CP-PACS Collaboration], Phys. Rev. D 76, 094506 (2007).

[55] X. Feng, K. Jansen, and D. B. Renner, Phys. Rev. D 83, 094505 (2011).

[56] S. Aoki et al. [CS Collaboration], Phys. Rev. D 84, 094505 (2011).

[57] C. B. Lang, D. Mohler, S. Prelovsek, and M. Vidmar, Phys. Rev. D 84, 054503 (2011).

[58] J. J. Dudek, R. G. Edwards, and C. E. Thomas [Hadron Spectrum Collaboration], Phys. Rev. D 87, 034505 (2013).

[59] C. Pelissier and A. Alexandru, Phys. Rev. D 87, 014503 (2013).

[60] G. S. Bali et al. [RQCD Collaboration], Phys. Rev. D 93, 054509 (2016).

[61] D. J. Wilson et al., Phys. Rev. D 92, 094502 (2015).

[62] J. Bulava et al., Nucl. Phys. B 910, 842 (2016).

[63] C. Alexandrou et al., Phys. Rev. D 96, 034525 (2017).

[64] B. Hu et al., Phys. Rev. D 96, 034520 (2017).

[65] C. Andersen, J. Bulava, B. Hörz, and C. Morningstar, Nucl. Phys. B 939, 145 (2019).

[66] R. García-Martín et al., Phys. Rev. D 83, 074004 (2011). 